\title{
Article \\ Plasmonic Elliptical Nanohole Arrays for Chiral Absorption and Emission in the Near-Infrared and Visible Range
}

\author{
Emilija Petronijevic*(D), Ramin Ghahri and Concita Sibilia \\ Department S.B.A.I., Sapienza Università di Roma, Via A. Scarpa 14, I-00161 Rome, Italy; \\ ramghahri@gmail.com (R.G.); concita.sibilia@uniroma1.it (C.S.) \\ * Correspondence: emilija.petronijevic@uniroma1.it
}

Featured Application: Plasmonic elliptical nanohole arrays coupled with emitting layers for nearinfrared and visible chiral sources.

Citation: Petronijevic, E.; Ghahri, R.; Sibilia, C. Plasmonic Elliptical

Nanohole Arrays for Chiral

Absorption and Emission in the Near-Infrared and Visible Range. Appl. Sci. 2021, 11, 6012. https:// doi.org/10.3390/app11136012

Academic Editor: Dimitrios Zografopoulos

Received: 21 May 2021

Accepted: 26 June 2021

Published: 28 June 2021

Publisher's Note: MDPI stays neutral with regard to jurisdictional claims in published maps and institutional affiliations.

Copyright: (c) 2021 by the authors. Licensee MDPI, Basel, Switzerland. This article is an open access article distributed under the terms and conditions of the Creative Commons Attribution (CC BY) license (https:/ / creativecommons.org/licenses/by/ $4.0 /)$.

\begin{abstract}
Chiral plasmonic nanostructures with tunable handedness-dependent absorption in the visible and infrared offer chiro-optical control at the nanoscale. Moreover, coupling them with emitting layers could lead to chiral nanosources, important for nanophotonic circuits. Here, we propose plasmonic elliptical nanohole arrays (ENHA) for circularly dependent near-infrared and visible emission. We first investigate broadband chiral behavior in an Au-ENHA embedded in glass by exciting it with plane waves. We then study the coupling of ENHA with a thin emitting layer embedded in glass; we focus on the emission wavelengths which provided high chirality in planewave simulations. Our novel simulation set-up monitors the chirality of the far-field emission by properly averaging a large set of homogeneously distributed, randomly oriented quantum sources. The intrinsic chirality of ENHA influences the circular polarization degree of the emitting layer. Finally, we study the emission dependence on the field distribution at the excitation wavelength. We demonstrate the chiral absorption and emission properties for Au-ENHA emitting in the nearinfrared range, and for Ag-ENHA which is excited in green range and emits in the Lumogen Red range. The simple geometry of ENHA can be fabricated with low-cost nanosphere lithography and be covered with emission gel. We thus believe that this design can be of great importance for tunable chiral nanosources.
\end{abstract}

Keywords: chirality; nanophotonics; plasmonics; nanohole arrays; nanosources

\section{Introduction}

Nanohole arrays (NHAs) are a class of nanostructured surfaces consisting of nanoscale voids fabricated in the surface of a material. Plasmonic NHAs can couple the incoming photons to plasma oscillations, which then result in surface plasmon polaritons (SPPs) [1-3]. These specific resonant electromagnetic excitations are strongly confined around the plasmonic NHA, and lead to electromagnetic field enhancement at the interface. SPPs can offering interesting phenomena such as sharp resonances, extraordinary optical transmission and extreme field enhancements. As such, they have been long investigated for applications including sensing, optical spectroscopy, surfaced enhanced Raman scattering etc. [4-9].

With the further development of nanotechnology, the introduction of symmetry breaking in plasmonic geometry offered additional, intriguing chiral effects at the nanoscale [10-12]. Namely, chiral nanostructures differently absorb circular polarization of the opposite handedness, leading to different optical response when excited by left and right circular polarization (LCP and RCP, respectively). This effect is known as circular dichroism (CD), and it has been long studied in the 3D nanostructures which mimic chiral shapes such as nanohelices [13-15], twisted bilayered metamaterials [16], folded eta-shaped metamaterials [17], and vertical nanowires asymmetrically covered by metal and excited at oblique 
incidence [18-20]. Moreover, chiral effects and CD can be obtained by the planarization of chiral nanostructures, which often simplifies the nanofabrication and the coupling with additional sensing or emitting material. Specifically, nanoholes with gammadionor helix-like chiral shapes provided chiral control in terms of transmission [21-23], or diffraction [24]. Moreover, the influence of the lattice asymmetry allows for the asymmetric, non-reciprocal transmission of circularly polarized light through misaligned SPPs in the plasmonic NHA [25]. More recently, we showed that the combination of lattice asymmetry and a simple elliptical nanohole shape can provide a chiral response in the near-infrared range. Namely, elliptical nanohole arrays (ENHA) with in-plane symmetry breaking can provide extremely high circular dichroism in the near-infrared range [26]. Furthermore, similar simple chiral geometries can be fabricated by cost- and time-efficient techniques such as nanosphere lithography (NSL) [27,28], and shadow sphere lithography [29]. Such passive nanostructures provide chiro-optical responses in transmission, reflection and absorption, thus showing the great potential for chiral sensing. On the other hand, similarly to the achiral NHA investigated in [30-32], ENHAs can be coupled with an emitting layer. Therefore, they can be of interest for both chiral sensing and chiral light sources.

In this work, we propose ENHAs for chiral emission in the near-infrared and visible ranges. Our numerical set-up combines two types of simulations: with plane-wave excitation, and with dipole excitation of the ENHA. Firstly, we report on the broadband chiral behavior of ENHAs for two plasmonic materials: gold (Au-ENHA) and silver (AgENHA); we embedded the 2D periodic ENHA in glass, and we excited them with LCP and RCP plane waves. Circular dichroism in absorption is mainly governed by the ENHA 2D periodicity, and it can be optimized in the near-infrared and visible domains. We then studied the coupling of the ENHA with a thin emitting layer embedded in glass above the plasmonic layer by exciting the ENHA with numerous electric dipole sources. Specifically, we investigated the chirality of far-field emission at wavelengths which provided high chirality in plane-wave simulations. For the Au-ENHA, we focused on the emission wavelengths of $740 \mathrm{~nm}$ and $940 \mathrm{~nm}$, which showed opposite chirality in the absorption; we show that the chirality also exhibits inversion in the emitted far-field. Next, we include the possibility of tuning the emitted chiral properties by engineering the excitation field confinement at shorter wavelengths. We demonstrate this effect for visible excitation and near-infrared emission of the Au-ENHA, and for green $(532 \mathrm{~nm})$ excitation and red $(630 \mathrm{~nm})$ emission of the Ag-ENHA, which is of interest for the commercially relevant Lumogen Red dye. Simulations of a large number of randomly oriented quantum emitters allow us to average the overall response, which makes our set-up adaptable for modelling real applications where emitters such as dye molecules are distributed in the refractive index matching material which covers the plasmonic layer. Moreover, the additional degree of freedom introduced by electromagnetic confinement at the excitation wavelength opens new perspectives in tunable efficient chiral light sources.

\section{Materials and Methods}

We investigated absorption and emission chirality by using two distinct simulation approaches in the commercially available finite difference time domain (FDTD) solver by Lumerical [33]. The first part of the work involved "passive" simulations of the chirooptical response of the ENHA previously optimized in [26]. The second part involved "active" simulations of distribution of dipoles within the dielectric layer above the ENHA. Finally, we combined the two approaches to account for the excitation influence on the emission chirality. Below, we present the two main simulation methods of this work.

Firstly, we simulated the broadband optical response of the ENHA in the range of 400-1000 nm using plane-wave excitation. In Figure 1a, we show the geometry of the ENHA, which is defined by the lattice parameter $a$, while $\mathrm{D}_{\mathrm{x}}$ and $\mathrm{D}_{\mathrm{y}}$ represent the short and the long nanohole diameters, respectively. Here, the FDTD domain is defined by periodic Bloch boundary conditions (BBC) in the xy-plane, while in the z-plane we define perfectly matched layers (PML). We take $t=100 \mathrm{~nm}$ as the thickness of the plasmonic ( $\mathrm{Au}$ 
or Ag) layer. This plasmonic layer lies on the semi-infinite dielectric substrate, and it is embedded in $300 \mathrm{~nm}$ of additional refractive index matching medium; in this way, the ENHA is surrounded by a dielectric medium with a refractive index of 1.45. The unit cell of the array is defined by the surface of $a$ and $a \sqrt{ } 3$, as NHAs with such hexagonal symmetry can be fabricated using the low-cost NSL technique (as in our previous experimental works). The nanohole has elliptical geometry, and the long ellipse axis is $15^{\circ}$ shifted with respect to the hexagonal symmetry, as has been previously optimized in [26]. For the Au-ENHA, we used the Au complex refractive index from the Lumerical material database; for the Ag-ENHA, we used experimental data from the ellipsometric measurements performed in our previous work [27]. In order to simulate circularly polarized plane-wave excitation, we combined two $\mathrm{x}$ - and $\mathrm{y}$-polarized broadband plane-wave sources with the same properties, except for the phase difference. The phase differences of $90^{\circ}$ and $-90^{\circ}$ correspond to LCP and RCP polarizations, respectively. The ENHA is excited under normal incidence from the top. Power monitors below the plasmonic layer and behind the source, detect transmission and reflection, respectively. Absorption is then calculated as $A_{i}=1-R_{i}-T_{i}$, where $\mathrm{i}=\mathrm{LCP}$ or $\mathrm{i}=\mathrm{RCP}$, and the final result of these passive simulations is the normalized circular dichroism in the absorption $C D_{A}$ :

$$
C D_{A}[\%]=100 \frac{A_{L C P}-A_{R C P}}{A_{L C P}+A_{R C P}} .
$$
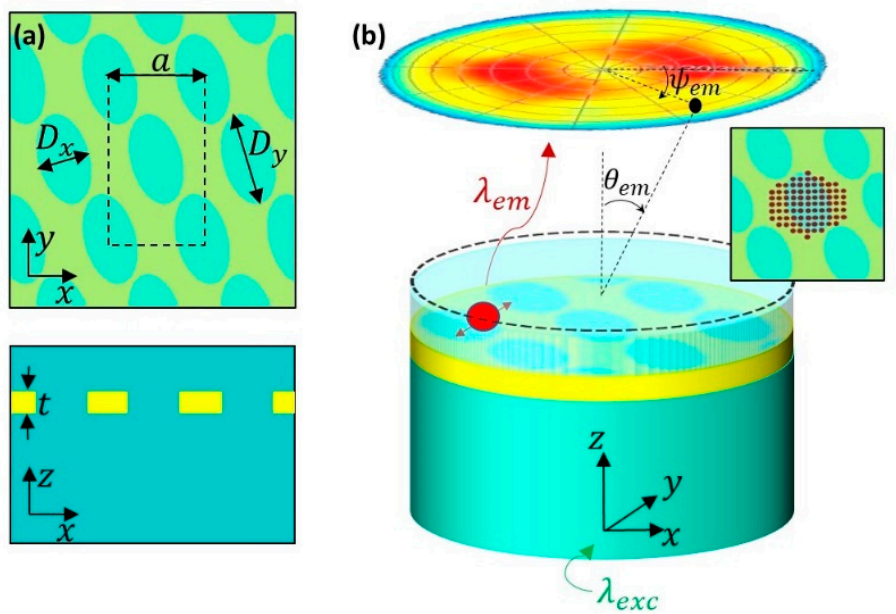

Figure 1. (a) xy- and xz-plane views of the ENHA, with geometric parameters. The dashed region in the top schematic shows the unit cell used in the "passive" simulations. (b) Simulation set-up for the "active" simulations; the randomly oriented dipole is positioned inside the embedding medium, $20 \mathrm{~nm}$ above the ENHA, and at a distance from the center which corresponds to one of the points in the inset. The dipole emits at a single wavelength $\lambda_{e m}$. The electromagnetic field at the excitation wavelength $\lambda_{\text {exc }}$ is further introduced as an additional tuning and optimization possibility.

While the passive properties of nanohole-based chiral materials have been previously studied, here we investigate the chiral properties of ENHAs coupled with the emitting layer. In the following, we will consider the light emission possibility of the ENHA coupled with dipole sources to be active. In this approach, a large FDTD domain surrounds more than 100 unit cells in the xy-plane, and it is defined by the PML boundaries in all six directions. A larger, non-periodic, FDTD volume is necessary in order to account for the periodic nature of the ENHA without introducing unnatural coherence [34]. An electric dipole oscillates as a point source, exciting the ENHA at $20 \mathrm{~nm}$ above the plasmonic layer. We show the schematic of such simulation in Figure 1b. The dipole emits at the single emission wavelength $\lambda_{e m}$, and it is defined by its orientation ( $\mathrm{x}-, \mathrm{y}-$, or $\mathrm{z}$-orientation), and position in the embedding layer. While we keep z position at $120 \mathrm{~nm}$ above the substrate, in the xy-plane we considered a homogeneous dipole distribution, which properly models 
dielectric layers doped with emitting materials. Next, we explain the novelty of the active simulation set-up for chiral emission, compared to previous, achiral nanostructures. It must be noted that all the chiral phenomena presented, arose from the coupling of an achiral dipole with a chiral nanostructure, as the dipole sources do not represent molecules which possess their own intrinsic chirality.

In the past, electromagnetic modelling followed experimental studies of fluorescence from emitting layers coupled to achiral NHA. The authors in $[30,31]$ reported on SPPmediated red light emission from circularly shaped nanoholes organized in a 2D periodic array with quadratic symmetry. The authors in [32] investigated low-cost achiral NHAs with hexagonal symmetry, coupled with $\mathrm{Er}^{3+}$ emitters. In these cases, the averaging of different dipole positions across the unit cell was simplified due to the in-plane symmetry of the achiral structures, allowing for the simulation of one quarter of the unit cell. Here, instead, the ENHA in-plane symmetry is broken by both the elliptical shape and the $15^{\circ}$ offset from the hexagonal symmetry lines. Therefore, the emitted characteristics need to be averaged over the complete distribution of the dipoles across the unit cell, which is done by properly modelling the homogeneous dipole distribution in the cell with hexagonal symmetry, as shown below.

The position of a dipole for a single simulation is defined by its $\Delta \mathrm{x}$ and $\Delta \mathrm{y}$ offset from the center of the FDTD domain; this domain, now defined by PML layers, has equal dimensions in the $\mathrm{x}$ - and $\mathrm{y}$-directions, thus preventing any additional asymmetry in the chirality calculations. Next, we focus on modelling suitable for real applications, where light emitters are homogeneously immersed in the host matrix in the vicinity of the nanostructures. In this case we consider emitters homogeneously distributed across the hexagon of the side $a / 2$, as shown in the inset of Figure $1 \mathrm{~b}$. The surface density of the dipoles across this hexagon is constant, while the hexagon itself encompasses one chiral object (i.e., an elliptical nanohole tilted from the symmetry of the hexagon). Therefore, the final averaging of the emitted field and its chirality will take into account both the dipoles close to the nanohole center and those close to the rims of the nanohole. After convergence testing, we chose the ensemble consisting of dipoles on the points defining the hexagon (shared between 3 hexagons), and two or three additional dipoles between these points, on the hexagon rims (shared between 2 hexagons). Considering the constant surface density, the total number of dipole positions equaled 85 , while the distance between two neighboring positions was held constant and equal to $(1 / 5)^{*}(\mathrm{a} / 2)$; the specific positions are marked as dots in the inset of Figure 1b. In this way, the homogeneity of the emitting material is ensured, while the polarization analysis of the simulation results reveals the overall chiral properties, which stem only from the chiral effects of the underlying nanostructure.

For each of the 85 possible positions, three simulations were run for each dipole orientation. The result of a single simulation was dipole radiation to the far-field, which was detected by a frequency-domain field-profile monitor. This near-field monitor was positioned in the air, $10 \mathrm{~nm}$ above the embedding medium, i.e., at $310 \mathrm{~nm}$ (dashed lines in Figure 1b); it encompasses the whole xy-plane of the FDTD region. The monitor collects the electromagnetic near-fields and decomposes them into a set of plane waves which propagate to the far-field. The near-to-far field projection results in a complex electromagnetic field which reaches the far-field hemisphere at a point defined by spherical coordinated $\theta_{\mathrm{em}}$ and $\Psi_{\mathrm{em}}$; there, it is decomposed into the LCP and RCP components by a Jones transfer matrix formalism. In this way, one can obtain $\mathrm{I}_{\mathrm{LCP}}$ and $\mathrm{I}_{\mathrm{RCP}}$, as well as the total intensity $\mathrm{I}_{\text {tot }}$ at each point on the hemisphere, which models the extrinsic chirality experiments where a quarter-wave plate and a linear polarizer were placed in the far-field $[35,36]$. The intensities from $3 \times 85$ simulations for all positions and orientations are then summed with weights $\eta$ which account for the dipoles, which are shared between two- or four-unit cells, depending on their position [32]:

$$
\bar{I}_{i}=\sum_{k=1}^{k=85} \eta_{k}\left(I_{i, k, x}+I_{i, k, y}+I_{i, k, z}\right)
$$


here, the subscript $i$ denotes the LCP or RCP field, or their sum $\left(\bar{I}_{L C P}, \bar{I}_{R C P}\right.$, and $\bar{I}_{t o t}$, respectively). The final result of these simulations is the circular dichroism in the emission $C D_{\mathrm{em}}$ :

$$
C D_{e m}[\%]=100 \frac{\bar{I}_{L C P}-\bar{I}_{R C P}}{\bar{I}_{L C P}+\bar{I}_{R C P}} .
$$

We performed convergence testing to ensure that simulation results did not depend on the FDTD volume [37]. As the simulation of single wavelength emission collects total, LCP and RCP far-fields after $3 \times 83$ single simulations, we saved time-cost of our simulations by means of the far-field filter. This Lumerical property is a parameter of near-to-far field transformation, set between 0 (which turns the filter off) and 1 [33]; it ensures that the electromagnetic fields at the very edges of the near-field monitor in the xy-plane went to zero, which translates to higher numerical stability and faster convergence. For all the following simulations, we opted for an FDTD domain of $36 \mu \mathrm{m}^{2}$ in the xy-plane, and a far-field filter of 0.1 .

As a final step, we combined the two simulation approaches to investigate the influence of the electromagnetic field at the excitation wavelength. Emission enhancement in plasmonic nanostructures is governed by both enhancement at the emission wavelength, and by the near-field confinement at the excitation wavelength [38]. The previous active simulations of 85 random dipoles account for the weights of dipoles at different positions in the xy-plane by considering the homogeneous medium and dipole sharing between the neighboring unit cells; in Figure $1 b, \eta=1$ for the dipoles inside the hexagon, $\eta=1 / 2$ for the dipoles on the hexagon margins, and $\eta=1 / 4$ for the two dipoles at the top and at the bottom. However, if the excitation laser leads to the enhancement and the inhomogeneous distribution of the electric field at the position of the dipoles, this can be approximated by a new weight factor, which now depends on both the position and the electric field confinement at excitation wavelength $\lambda_{\text {exc }}$. We extracted this factor by performing passive single-wavelength simulation at $\lambda_{\text {exc }}$, and by collecting the electric field intensity at the points of the dipole positions at $\mathrm{z}=120 \mathrm{~nm}$. Finally, we normalized the emission of the dipoles as:

$$
\bar{I}_{i}=\sum_{k=1}^{k=85} \eta\left(\left|\vec{E}\left(k, \lambda_{\text {exc }}\right)\right|^{2}\right)_{k}\left(I_{i, k, x}+I_{i, k, y}+I_{i, k, z}\right)
$$

\section{Results and Discussion}

Below, we present step by step of the design towards tunable chiral sources based on ENHAs and emitting layers. We start from the passive simulations of $\mathrm{CD}_{\mathrm{A}}$ in $\mathrm{Au}$ - and Ag-ENHA. Then, we investigate the active simulations of single dipoles distributed as the emitting layer for the Au-ENHA, and we investigate their average influence on the chiral emission in the near-infrared range. Finally, we investigate the Ag-ENHA with polarization-dependent excitation in the green range, and emission in the red visible range.

\subsection{Passive Simulations}

In our previous numerical work, we showed that chiral absorption in ENHA arises due to the different coupling of LCP- and RCP- excited SPPs with elliptical nanoholes tilted from the lines of the 2D array symmetry [26]. We optimized the ENHA with $a=500 \mathrm{~nm}$ in terms of $\mathrm{D}_{\mathrm{x}}, \mathrm{D}_{\mathrm{y}}$, and the tilt angle $\phi$; we found that the optimum $\mathrm{CD}_{\mathrm{A}}$ (reaching almost $84 \%$ ) was around $745 \mathrm{~nm}$ for $\phi=15^{\circ}, D_{x}=280 \mathrm{~nm}$, and $D_{y}=500 \mathrm{~nm}$. These ENHA were standing upon glass substrates in air; therefore, their spectral features and $\mathrm{CD}_{\mathrm{A}}$ were expected to red-shift with an increase of the refractive index of the surrounding medium.

Here, we started from the previously optimized ENHA geometry, and embedded it in the refractive index-matching layer, which served as a dielectric matrix for the emitters in the active simulations. In a hexagonal lattice, the ENHA tilt angle of $\phi=15^{\circ}$ always provides the highest $C D_{A}$, so we kept this parameter constant. We investigated $C D_{A}$ dependence on various separate geometric parameters (not shown here), and conclude 
that its spectral features mainly depend on lattice periodicity, which defines the light coupling to the SPP modes. Therefore, here we show the results of the parameter sweep over the periodicity range of $a=350-550 \mathrm{~nm}$, while we scale the nanohole surface in the unit cell according to the previous optimization, i.e., $\mathrm{D}_{\mathrm{x}}=280^{*} a / 500$, and $\mathrm{D}_{\mathrm{y}}=a$. We investigate this range as it is of interest for future fabrication; namely, the starting diameter of commercial polystyrene nanospheres determines the hexagonal array periodicity in the NSL [32]. Figure 2a,b show spectral maps of Au-ENHA absorption dependence for the LCP and RCP excitations, respectively. Spectral features linearly shift with $a$, as is expected for the linearity of the phase matching condition of SPPs. Below $540 \mathrm{~nm}$, absorption for both excitations increases in the same manner due to the intraband absorption in Au. For LCP excitation and lattices around $a=500 \mathrm{~nm}$ and larger, there is one main plasmonic maximum in the near-infrared. Changing the excitation to $\mathrm{RCP}$, the quality factor of this maximum increases, and other plasmonic features at larger wavelengths appear. This different spectral behavior leads to a high $\mathrm{CD}_{\mathrm{A}}$ in the near-infrared range, with two prominent branches in the opposite $\mathrm{CD}_{\mathrm{A}}$ signs, as seen in Figure 2c. Specifically, for $a=500 \mathrm{~nm}, \mathrm{CD}_{\mathrm{A}}$ reaches around $50 \%$ at $740 \mathrm{~nm}$, and inverts the behavior at $940 \mathrm{~nm}$. This peculiar behavior of ENHA absorption influences the coupling with the emitting layer; therefore, these two wavelengths will be considered for dipole emission further in the manuscript. Moreover, we note that under $600 \mathrm{~nm} \mathrm{CD}_{\mathrm{A}}$ vanishes because of the absence of the plasmonic coupling, and, eventually, $\mathrm{Au}$ intraband absorption. Finally, one can note that the maximum $\mathrm{CD}_{\mathrm{A}}$ branches red-shifted because of the superstrate, while their absolute value decreased with respect to the design previously optimized in [26]. In this manuscript, we focus on the investigation of the chiral absorption and emission phenomena which take place in the previously designed ENHA, embedded in glass. However, this design is scalable and can be easily re-optimized for specific emission and excitation wavelengths. Once the wavelength of maximum $\mathrm{CD}_{\mathrm{A}}$ is defined, one can perform multiparameter optimization of parameters $a, \mathrm{D}_{\mathrm{x}}, \mathrm{D}_{\mathrm{y}}$, and $\mathrm{t}$, to get the highest contrast of RCP and LCP absorption at those wavelengths by means of particle-swarm algorithm [39].
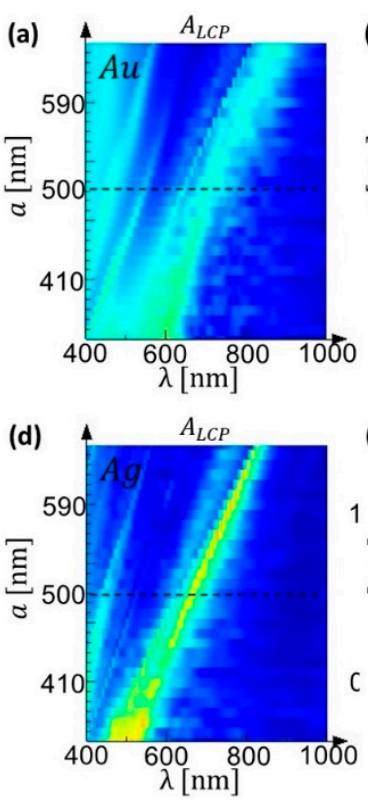

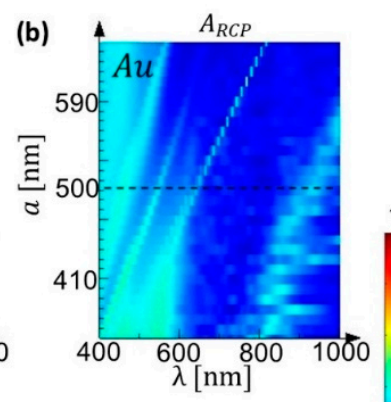

(e)

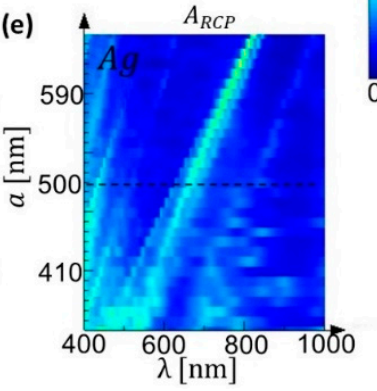

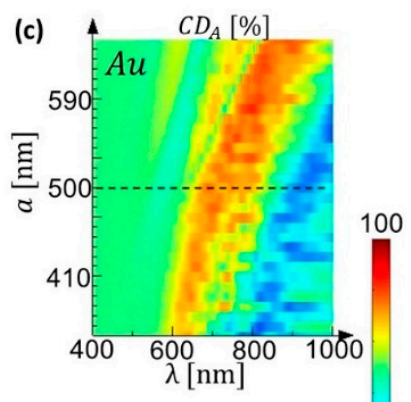

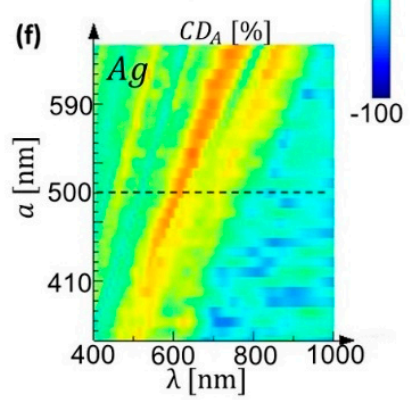

Figure 2. Passive simulations of chiral absorption in Au-ENHA and Ag-ENHA as dependences on lattice parameter $a$. ENHA diameters are scaled as $\mathrm{D}_{\mathrm{x}}=280^{*} a / 500, \mathrm{D}_{\mathrm{y}}=a$, while the angle offset from the hexagonal symmetry is held at $15^{\circ}$. (a) $\mathrm{A}_{\mathrm{LCP}}$, (b) $\mathrm{A}_{\mathrm{RCP}}$, and (c) $\mathrm{CD}_{\mathrm{A}}$ for the Au-ENHA; (d) $A_{\mathrm{LCP}},(\mathbf{e}) \mathrm{A}_{\mathrm{RCP}}$, and (f) $\mathrm{CD}_{\mathrm{A}}$ for the Ag-ENHA.

In Figure $2 \mathrm{~d}-\mathrm{f}$, we repeat the same $a$ sweep when the plasmonic materials is $\mathrm{Ag}$, by keeping all the geometric features equal as before. As expected, there was a blue-shift of 
all spectral features compared to the Au-ENHA due to the higher plasma frequency of $\mathrm{Ag}$. The branches of $\mathrm{CD}_{\mathrm{A}}$ now reach shorter visible wavelengths, where the Ag-ENHA can be excited e.g., with a green laser. Therefore, while Au-ENHA can be used for the near-infrared, while Ag is more suitable for the visible range.

\subsection{Active Simulations}

The purpose of this section is to investigate the influence of the $\mathrm{CD}_{\mathrm{A}}$ on $\mathrm{CD}_{\mathrm{em}}$. Therefore, we chose the Au-ENHA with $a=500 \mathrm{~nm}$, and we applied active simulations for two typical wavelengths. Figure 3 plots the typical results of our numerical method for a randomly oriented dipole situated at $\Delta \mathrm{x}=0, \Delta \mathrm{y}=\mathrm{a} / 2$, and $\Delta \mathrm{z}=120 \mathrm{~nm}$, emitting at $740 \mathrm{~nm}$ (top), and $940 \mathrm{~nm}$ (bottom). The simulation of one dipole position contained three simulation runs (for $\mathrm{x}-, \mathrm{y}-$, and $\mathrm{z}$-orientation), where the far-field matrices of the total, $\mathrm{LCP}$, and RCP far-field emission were saved, and eventually averaged. At both wavelengths, total intensity is highly irregular due to ENHA chirality and the offset of the dipole with respect to the unit cell center. At longer wavelengths, the overall total light emission is directed towards larger angles, and with less intensity, as is expected due to the coupling with the lattice $[30,31]$. When the total field is decomposed into opposite circular polarizations, we notice strong chirality, especially at $940 \mathrm{~nm}$ : there is an absence of RCP compared to LCP in the central part of the far-field hemisphere. However, a single dipole position cannot account for the chiral properties of the emitting material, situated in a matrix material above the ENHA. We believe that there are two main reasons we need to simulate the emission of large number of dipoles, before taking their average intensities in Equation (2), and calculating the $\mathrm{CD}_{\mathrm{em}}$. Firstly, total intensity differed strongly for the dipoles situated on the rims and in the nanohole center, as the local density of states in plasmonic nanostructures strongly depend on spatial confinement. Secondly, in achiral NHAs, two dipoles situated at opposite sides of the NHA give opposite $\mathrm{CD}_{\mathrm{em}}$, according to extrinsic chirality, whereas their average gives zero $\mathrm{CD}_{\mathrm{em}}$. ENHAs are intrinsically chiral, so we expect that the interplay of these two phenomena would give a non-zero chirality in the averaged emitted field.

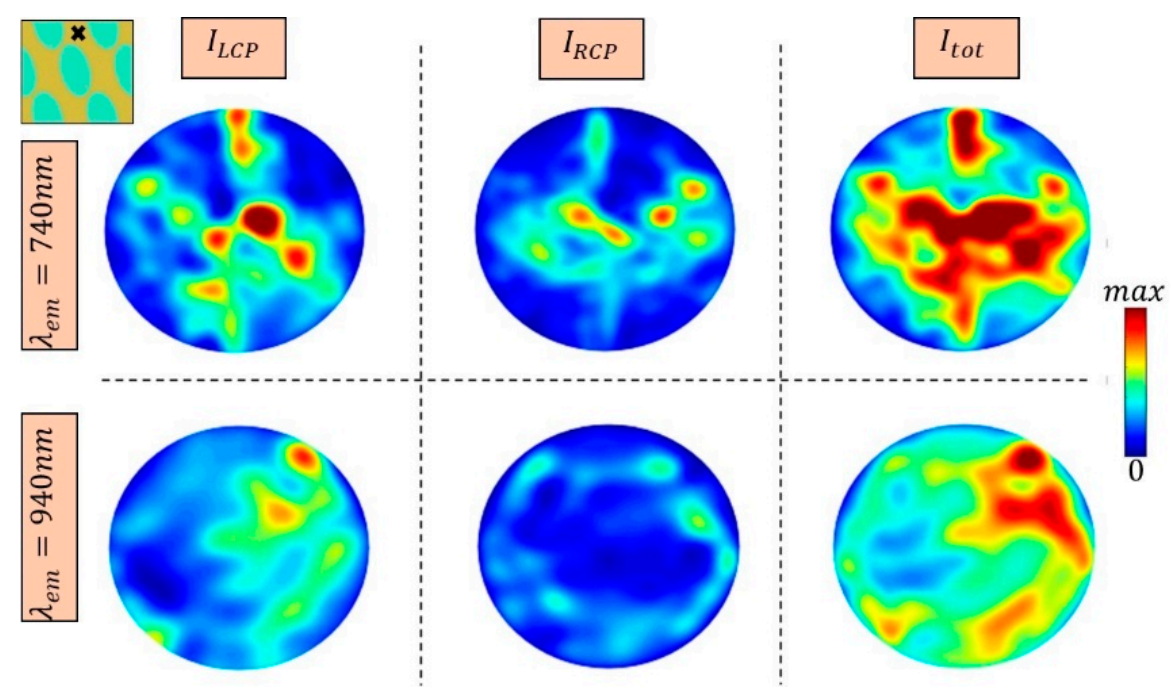

Figure 3. Simulation results for a randomly oriented dipole positioned at $\Delta x=0, \Delta y=a / 2$, and $\Delta \mathrm{z}=120 \mathrm{~nm}$, emitting at $740 \mathrm{~nm}$ (top), and $940 \mathrm{~nm}$ (bottom); we performed three simulation runs for each dipole orientation, extract LCP, RCP and total far-field intensity for each simulation, and then took the average.

We investigate the previous point by calculating the $\mathrm{CD}_{\mathrm{em}}$ from typical dipole positions in the unit cell (over the hexagon presented in the inset of Figure 1b). In Figure 4, it is evident that the dipoles situated between the nanoholes (corners of the figure), led to extremely high chirality close to the normal incidence. As the dipole position approaches the 
unit cell center, the circular dichroism drops. This can be of interest for light nanosources such as quantum dots, which can be precisely positioned at the points which exhibited high $C D_{\text {em }}$. On the other side, for emitting layers with fluorescent dopants, the single position-chiral behavior can give guidelines for the engineering of the excitation control, as will be shown later.

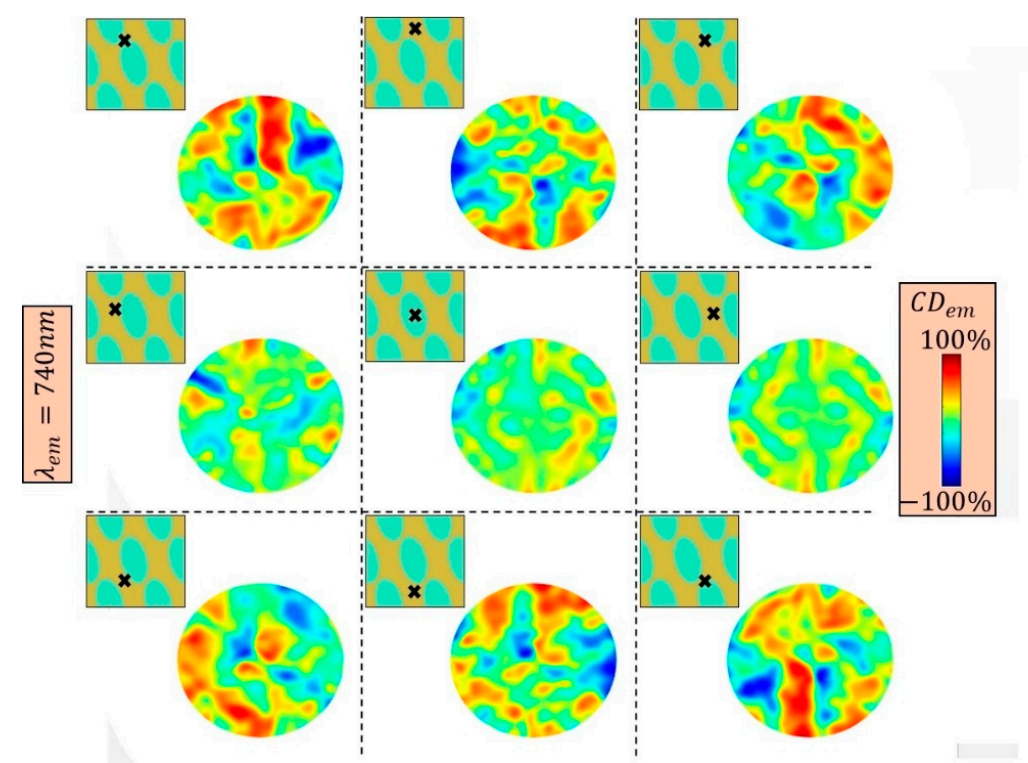

Figure 4. $\mathrm{CD}_{\mathrm{em}}$ maps for different dipole positions on the Au-ENHA, at $740 \mathrm{~nm}$.

In Figure 5, we show chirality of typical dipole positions for the emission at $940 \mathrm{~nm}$. Even though emission intensity was lower (Figure 3), for most positions there was a strong positive chirality in the central part of the hemisphere, which is wider and more pronounced with respect to Figure 4 . Again, $\mathrm{CD}_{\mathrm{em}}$ decreases towards the dipoles closer to the ENHA center.

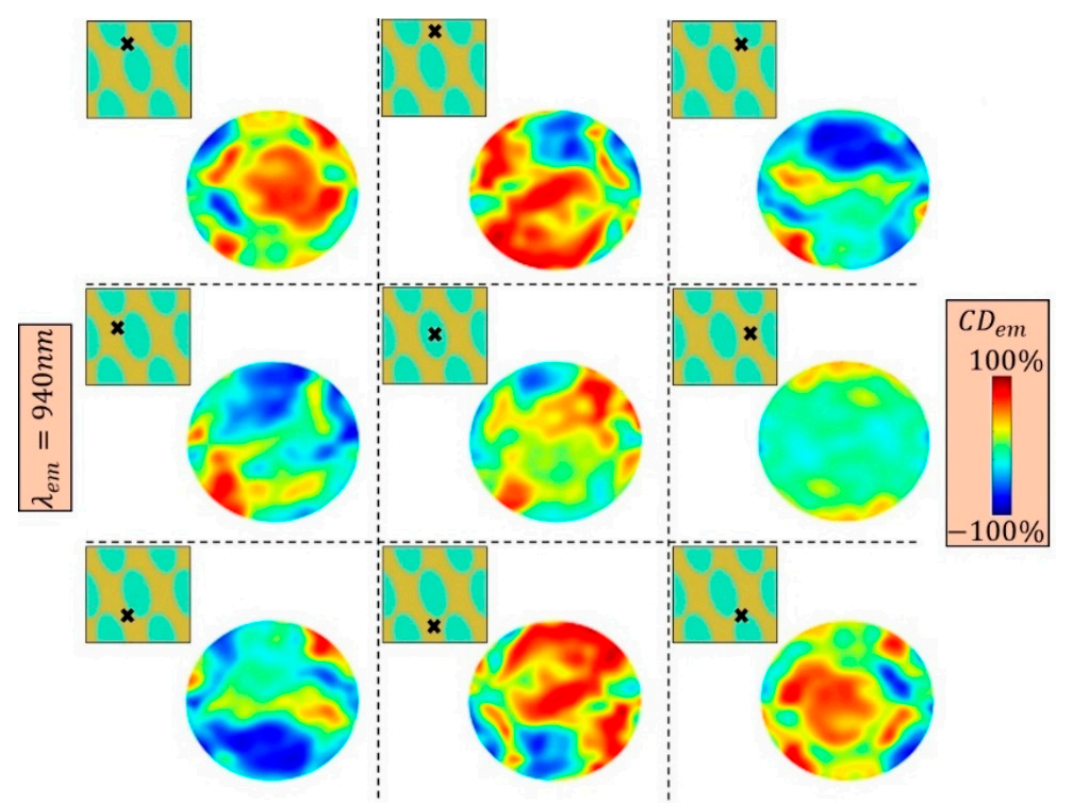

Figure 5. $\mathrm{CD}_{\mathrm{em}}$ maps for different dipole positions for the Au-ENHA, at $940 \mathrm{~nm}$.

Finally, we apply Equation (3) to calculate the total, LCP, and RCP fields emitted by the ensemble of 85 dipoles, Figure 6 . Here we consider the dipole weights with respect to the shared geometry between the neighboring cells, while we do not consider confinement 
at the excitation wavelength. As is expected, higher emission at $740 \mathrm{~nm}$ corresponds to higher confinement of this wavelength at $120 \mathrm{~nm}$. Moreover, at $940 \mathrm{~nm}$ the light was directed towards larger angles in the far-field, in agreement with diffraction-governed optical phenomena. More importantly, in the direction of the maximum emission at $740 \mathrm{~nm}$ $\left(\Psi_{\mathrm{em}} \sim 0^{\circ}\right)$ the overall $\mathrm{CD}_{\mathrm{em}}$ switched the sign between the two wavelengths, just as in $\mathrm{CD}_{\mathrm{A}}$. Therefore, passive ENHA chiral properties directly influence their coupling with the achiral emitting layer situated in the index matching layer. Absolute $\mathrm{CD}_{\mathrm{em}}$ values reached $20 \%$ and $30 \%$ for $740 \mathrm{~nm}$ and $940 \mathrm{~nm}$, respectively.

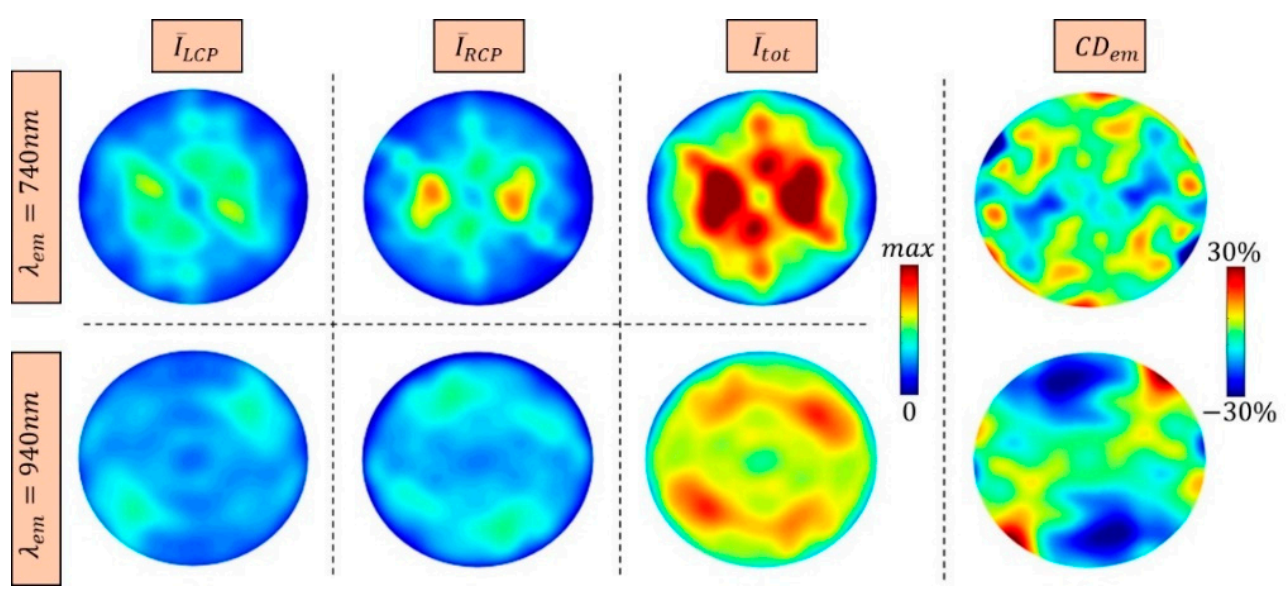

Figure 6. $\overline{\mathrm{I}}_{\mathrm{LCP}}, \overline{\mathrm{I}}_{\mathrm{RCP}}, \overline{\mathrm{I}}_{\mathrm{tot}}$, and $\mathrm{CD}_{\mathrm{em}}$ maps for the ensemble of 85 homogeneously distributed randomly oriented dipoles for the Au-ENHA, at $740 \mathrm{~nm}$ (top), and $940 \mathrm{~nm}$ (bottom).

\subsection{Influence of Excitation}

Figures 4 and 5 show that dipole position strongly influences chiral emission in $C_{\text {em }}$ and its intensity. An additional way to boost and tune this performance is to add another degree of freedom: electromagnetic confinement at the excitation wavelength. Apart from controlling the emission direction, polarization and intensity, plasmonic nanostructures can boost absorption in the emitting material by controlling its excitation rate $\gamma_{\mathrm{ab}}$, as $\gamma_{a b} \sim\left|\vec{\mu}_{a b} \vec{E}_{\text {exc }}\right|^{2}$, where $\vec{\mu}_{a b}$ is the absorption dipole moment, and $\vec{E}_{\text {exc }}$ is the electric field at the excitation wavelength at the dipole position [40]. Different plasmonic systems have been used to optimize molecule fluorescence by means of near field enhancement at the excitation wavelength [38,40-44]. In such systems, excitation field properties such as wavelength, incidence angle, and polarization can be engineered to enhance electromagnetic confinement in the parts of the nanostructure volume filled with the emitting material.

Next, we demonstrate the influence of excitation (wavelength and polarization) on the emitting chiral properties of Au-ENHA with $a=500 \mathrm{~nm}$. We choose to investigate $\lambda_{\text {exc }}=532 \mathrm{~nm}$, which is a common green laser used for the excitation of emitters with plasmonic nanostructures [45], and $\lambda_{\text {exc }}=740 \mathrm{~nm}$, where Au-ENHA shows high $\mathrm{CD}_{\mathrm{A}}$. As our ENHAs are chiral over a broad spectral range, we expect that the switching of LCP to RCP in the excitation influences emitted field intensity and chirality. We started from the passive behavior of the Au-ENHA at single wavelengths by exciting the ENHA from the bottom (positive z-direction), with RCP or LCP (top and bottom panels of Figure 7, respectively). The electric field intensity at the excitation wavelength $\left|\vec{E}_{\text {exc }}\right|^{2}$ was detected by two field-profile monitors over the unit cell in the $x z$ cross-section, centered at $y=0 \mathrm{~nm}$, and in the xy cross-section, centered at $z=120 \mathrm{~nm}$ (note that this is the same position as the dipole ensemble in the active simulations). This intensity was normalized to the incident field intensity $\left|\vec{E}_{0}\right|^{2}$, with $E_{0}=1 \mathrm{~V} / \mathrm{m}$. As can be appreciated from Figure $2 \mathrm{a}, \mathrm{b}$, at $\lambda_{\text {exc }}=532 \mathrm{~nm}$ there was no increased coupling with the ENHA, hence it did not possess chiral behavior in the absorption (green area in Figure 2c). Indeed, Figure 7a shows that for both LCP and RCP excitation, the electric field was not confined to the metallic layer, and 
did not overlap with the emitter position (low confinement across the whole xy-monitor). However, there was a slight difference in its distribution, with the switch of the excitation handedness. On the other side, Figure $7 \mathrm{~b}$ shows high coupling with the ENHA, which was greatly enhanced for the excitation switching from RCP to LCP. This is in agreement with the strong chirality at this wavelength, as seen in the orange region of Figure 2c.
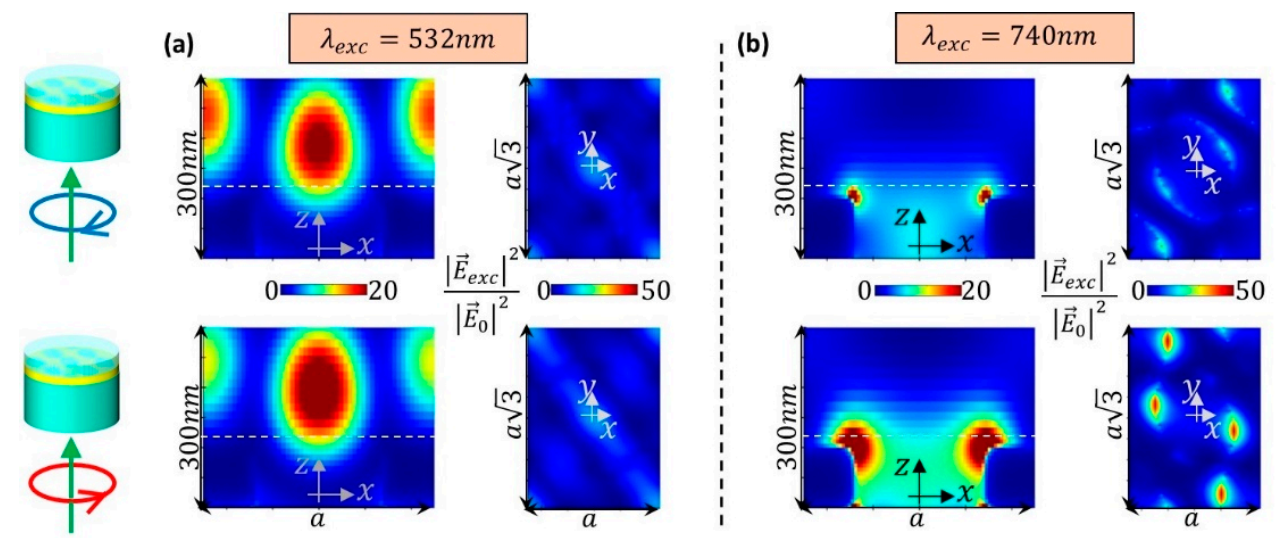

Figure 7. Passive simulations of the electric field intensity enhancement for the Au-ENHA, when its excited with RCP (top), or LCP (light) from the bottom. We plot normalized electric field intensity in two-unit cell cross-sections, detected by $\mathrm{xz}$-monitor (centered at $\mathrm{y}=0 \mathrm{~nm}$ ), and $\mathrm{xy}$-monitor (situated at $\mathrm{z}=120 \mathrm{~nm}$ ), at (a) $532 \mathrm{~nm}$, and (b) $740 \mathrm{~nm}$.

For each of simulations presented in Figure 7, we save the intensities of each field component in the points defined in the inset of Figure $1 \mathrm{~b}$. As was expected for the confinement with plasmonic nanohole arrays, the main field component for each excitation was $E_{z}$ (not shown here); this means that the z-oriented dipoles standing on the nanohole rims will enter the sum in Equation (4), with significantly higher weight. Next, we calculated the LCP and RCP light emission by averaging all dipole positions and orientations with this additional weight factor from the passive simulations, thus accounting for the excitation electric field intensity at the position of the dipoles. To demonstrate strong influence of the excitation on both intensity and chirality of emission, we investigated how light emitted at $\lambda_{e m}=940 \mathrm{~nm}$ depends on the excitation wavelengths $\lambda_{\text {exc }}=532 \mathrm{~nm}$ and $\lambda_{\text {exc }}=740 \mathrm{~nm}$, and on the choice of RCP and LCP, as shown in Figure 8. Since the results are plotted with the same color bar, we note a strong enhancement in the case of LCP excitation at $740 \mathrm{~nm}$. This is a consequence of the high field confinement at the position of the dipole ensemble, as seen in Figure $7 \mathrm{~b}$. The emission intensities are $\sim 5$ times lower for the excitation at $532 \mathrm{~nm}$, compared to $740 \mathrm{~nm}$, while the resulting emitted field distribution shows distinguishable behavior. Namely, at $532 \mathrm{~nm}$ excitation, we notice a slight enhancement of both $\overline{\mathrm{I}}_{\mathrm{LCP}}$ and $\overline{\mathrm{I}}_{\text {RCP }}$ when its handedness is switched from RCP to LCP, as seen in Figure 8a. At $740 \mathrm{~nm}$ excitation, the LCP and RCP emitted components drastically change in distribution and increase in intensity from the excitation change from RCP to LCP, as seen in Figure $8 b$. This is because the Au-ENHA is strongly chiral at this excitation wavelength, with a pronounced change of the electromagnetic confinement over the ensemble (see the xy cross-sections in Figure $7 \mathrm{~b}$ ).

Finally, Figure 9 plots the resulting chiral emission properties for different combinations of the excitation and emission wavelengths, and excitation handedness. We first investigated Au-ENHA, with $a=500 \mathrm{~nm}$. In the left panel of Figure 9, we show the $\mathrm{CD}_{\mathrm{em}}$ calculated from the maps in Figure 8a; we notice that even a slight change of the excitation field distribution (by changing it handedness) can influence the overall map. $C D_{\mathrm{em}}$ is rather low close to the normal incidence, but increases in the far-field points which do not have high emission intensity. Therefore, such a configuration is not suitable for excitation. Moving to $\lambda_{\text {exc }}=740 \mathrm{~nm}$, which strongly couples to the Au-ENHA, the emitted intensity increases and the emission becomes highly tunable by means of excitation (Figure $8 \mathrm{~b}$ ). 
Indeed, in the middle panel of Figure 9, the maximum absolute value of $\mathrm{CD}_{\mathrm{em}}$ increases from $30 \%$ in Figure 6, to 50\%. As expected, chirality at the excitation wavelength plays an important role in tailoring the $\mathrm{CD}_{\mathrm{em}}$ : it increases in absolute value for LCP excitation, and it switched the sign for angles close to normal incidence. Finally, we consider a combination which could be of interest for the ENHA coupled with Styryl 8 dye, which emits around $740 \mathrm{~nm}$, and which can be excited at $633 \mathrm{~nm}$ [30,31]. In the right panel of Figure 9, we see that an absolute $\mathrm{CD}_{\mathrm{em}}$ of $\sim 20 \%$ can be obtained at in the center of the far-field for the LCP excitation.
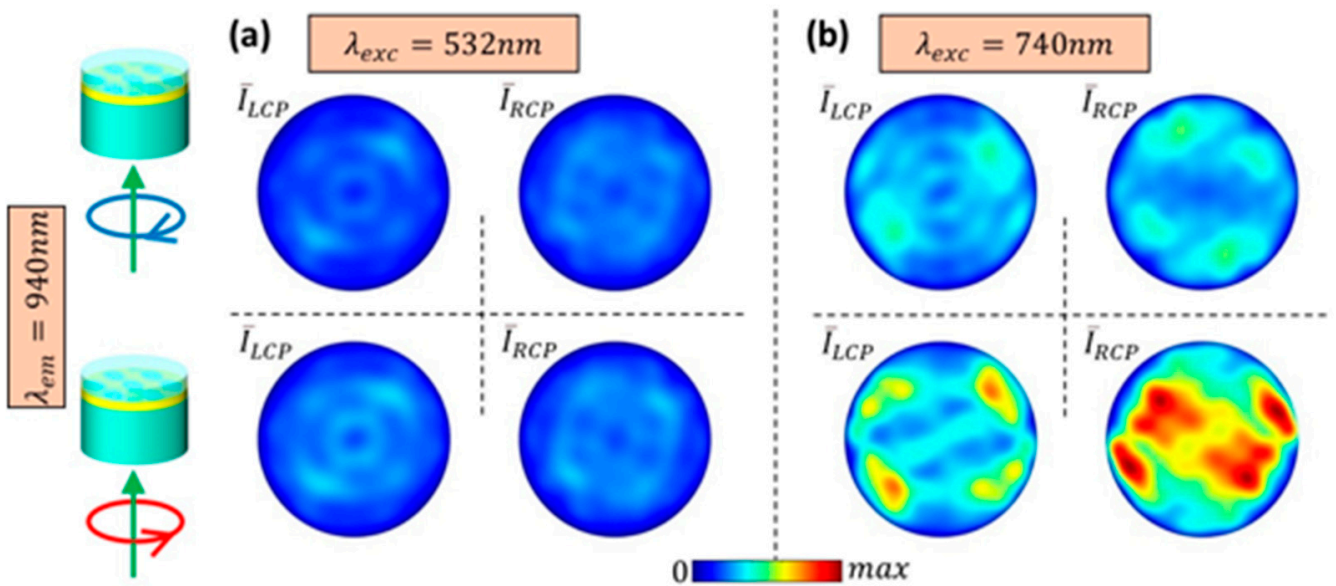

Figure 8. $\overline{\mathrm{I}}_{\mathrm{LCP}}$ and $\overline{\mathrm{I}}_{\mathrm{RCP}}$ far-field maps of the Au-ENHA emitting at $940 \mathrm{~nm}$, when it is excited with RCP (top) and LCP (bottom), at the wavelengths (a) $\lambda_{\text {exc }}=532 \mathrm{~nm}$, and (b) $\lambda_{\text {exc }}=740 \mathrm{~nm}$.

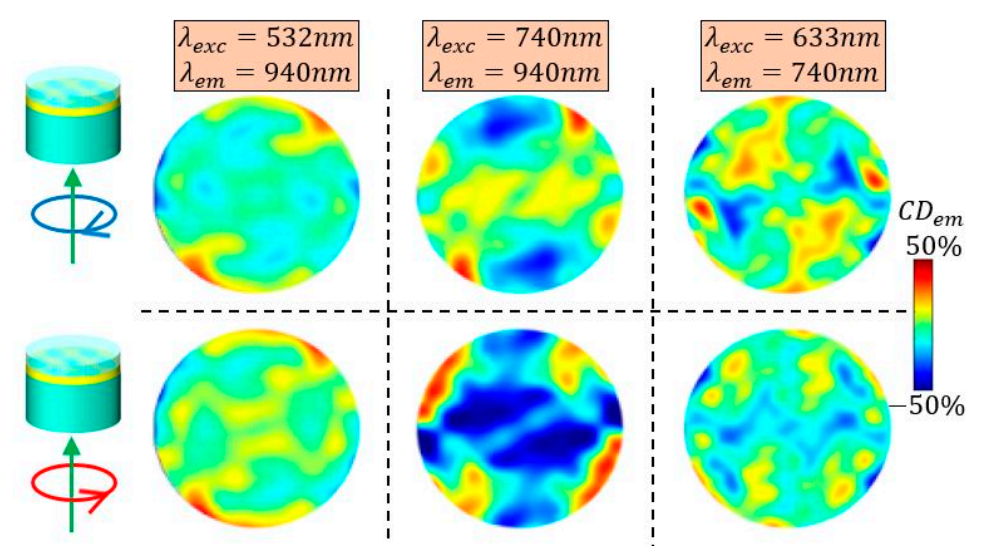

Figure 9. $\mathrm{CD}_{\mathrm{em}}$ for RCP (top) and LCP (bottom) excitations of the Au-ENHA for $=\lambda_{\text {exc }}=532 \mathrm{~nm}$, $\lambda_{e m}=940 \mathrm{~nm}$ (left), $\lambda_{e x c}=740 \mathrm{~nm}, \lambda_{e m}=940 \mathrm{~nm}$ (middle), and $\lambda_{\text {exc }}=633 \mathrm{~nm}, \lambda_{\text {em }}=740 \mathrm{~nm}$ (right).

Next, we considered Ag-ENHA with $a=510 \mathrm{~nm}$, coupled with a fluorescent ensemble excited at $532 \mathrm{~nm}$ and emitting at $630 \mathrm{~nm}$. We choose these wavelengths as they can be of interest for Lumogen Red dye, which has the typical absorption and emission spectra shown in Figure 10a [45]. In this case, both the excitation and emission wavelengths lie in the positive $\mathrm{CD}_{\mathrm{A}}$ branch of our nanostructure (Figure $2 \mathrm{f}$ ). In the inset of Figure 10b, we show the normalized electric intensity over the xy cross-section at $120 \mathrm{~nm}$. Compared to the Au-ENHA (Figure 7a), RCP and LCP distribution strongly differed. This means that RCP excitation at $532 \mathrm{~nm}$ enhanced the emission of the dipoles situated in the middle of the nanohole. LCP excitation, on the contrary, increased the contribution of the dipoles on the rims of the nanohole. Therefore, the resulting emitted field shows a dramatic change in distribution for the excitation handedness change, as seen in Figure 10b. 


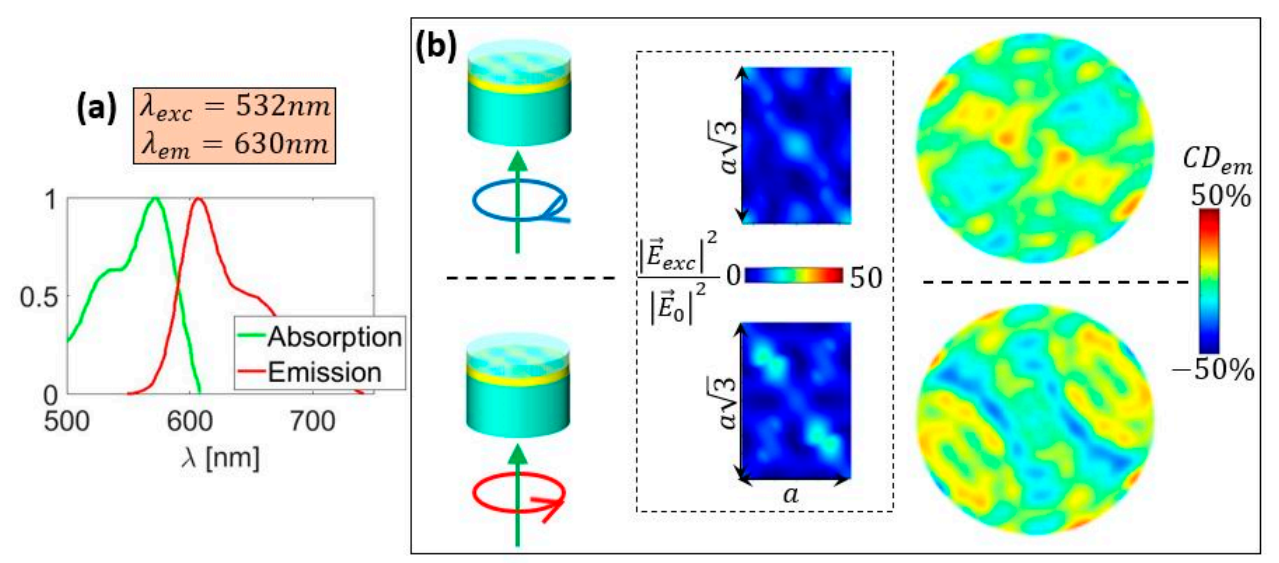

Figure 10. (a) Typical absorption and emission spectra for Lumogen Red dye [45]. (b) $\mathrm{CD}_{\mathrm{em}}$ for RCP (top) and LCP (bottom) excitations of Ag-ENHA excited at $\lambda_{\text {exc }}=532 \mathrm{~nm}$, and emitting at $\lambda_{e m}=630 \mathrm{~nm}$; the inset shows the xy cross-sections of the electric field intensity, as in Figure 7.

$\mathrm{CD}_{\text {em }}$ can be enhanced and directed differently in the far-field by changing the polarization of the excitation. The rich chiral behavior of ENHAs can be used to optimize the desired far-field properties and chirality for a specific application in many ways. Take as an example an ENHA coupled with molecules which emit at $940 \mathrm{~nm}$. If one needs high $\mathrm{CD}_{\mathrm{em}}$ emitted directly to the far-field center $\left(\theta_{\mathrm{em}} \sim 0^{\circ}, \Psi_{\mathrm{em}} \sim 0^{\circ}\right)$, the excitation can be tuned to enhance the dipoles on the upper left and lower right positions in the unit cell, as seen in Figure 5. Another requirement can be a high contrast ratio between the $C D_{e m}$ with excitation switching from RCP to LCP. As an example, the far-field $C_{\mathrm{em}}$ distributions in Figure $9 \mathrm{~b}$ show that this is possible for light directed in $\theta_{\mathrm{em}} \sim 10^{\circ}$ cone, and it can be further optimized. Therefore, proper engineering of the field confinement at the excitation wavelength can enhance the contribution of the dipoles which give the highest $\mathrm{CD}_{\mathrm{em}}$ of the same sign, while the change to their polarization can tune the desired property in the emitted far-field. Moreover, the position of the ensemble in the z-direction can be matched with the high chiral properties. Finally, excitation angle of incidence plays can be used to couple the incident excitation light to the resonance of the nanostructures $[41,43,45,46]$; e.g., in Figure 8a, this would lead to higher intensity of the emitted light and to an additional degree of freedom in tuning the resulting $\mathrm{CD}_{\mathrm{em}}$ (Figure 9, left). Finally, the optimization of the emission chiral properties by the excitation of the polarization state, wavelength, and direction will be the subjects of future work.

\section{Conclusions}

In this work, we proposed the coupling of simple, planar, chiral nanohole arrays with an ensemble of emitting material, for chiral emission in the near-infrared and visible ranges. Our numerical method treats the chiral properties of a large number of randomly oriented dipoles, which were homogeneously positioned in a refractive index matching matrix above the ENHA. The chiral properties of the emitted far-field strongly depend on the chiral properties of the plasmonic substrate. Moreover, the excitation of the dipoles at shorter wavelengths can tune the electromagnetic field confinement in the volume of the emitting layer. As such, the excitation field can be used to enhance the emission of specific dipoles, and to control it by selecting proper excitation wavelength and polarization. Thus, it provides additional degree of freedom in the tuning of the overall emission in terms of the degrees of intensity and circular polarization. Even though our design involves a simple elliptical nanohole geometry in an Au or Ag layer, it provides rich chiral behavior in both absorption and emission. Therefore, we strongly believe that the ENHA coupled with emitting layers can be optimized towards tunable efficient chiral nanosources. 
Author Contributions: Conceptualization, E.P. and C.S.; methodology, E.P. and C.S.; software, E.P. and R.G.; validation, C.S.; formal analysis, R.G.; investigation, E.P. and R.G.; resources, C.S.; data curation, R.G.; writing-original draft preparation, E.P. and R.G.; writing-review and editing, C.S.; visualization, R.G.; supervision, C.S.; project administration, C.S.; funding acquisition, C.S. All authors have read and agreed to the published version of the manuscript.

Funding: This research received no external funding.

Institutional Review Board Statement: Not applicable.

Informed Consent Statement: Not applicable.

Acknowledgments: In this section, you can acknowledge any support given which is not covered by the author contribution or funding sections. This may include administrative and technical support, or donations in kind (e.g., materials used for experiments).

Conflicts of Interest: The authors declare no conflict of interest.

\section{References}

1. Maier, S.A. Plasmonics: Fundamentals and Applications; Springer Science \& Business: Media, Germany, 2007.

2. García de Abajo, F.J. Colloquium: Light scattering by particle and hole arrays. Rev. Mod. Phys. 2007, 79, 1267. [CrossRef]

3. Polman, A.; Atwater, H.A. Plasmonics: Optics at the nanoscale. Mater Today 2005, 8(1), 56. [CrossRef]

4. Couture, M.; Liang, Y.; Poirier Richard, H.-P.; Faid, R.; Peng, W.; Masson, J.-F. Tuning the 3D plasmon field of nanohole arrays. Nanoscale 2013, 5, 12399. [CrossRef]

5. Maradudin, A.; Sambles, J.R.; Barnes, W.L. Modern Plasmonics, Volume 4, 1st ed.; Elsevier Science: Amsterdam, The Netherlands, 2014.

6. Smolyaninov, I.; Zayats, A. Near-field photonics: Surface plasmon polaritons and localized surface plasmons. J. Opt. A Pure Appl. Opt. 2003, 5, S16.

7. Barnes, W.L.; Dereux, A.; Ebbesen, T.W. Surface plasmon subwavelength optics. Nature 2003, 424, 824. [CrossRef] [PubMed]

8. Salomon, L.; Grillot, F.; Zayats, A.V.; de Fornel, F. Near-Field Distribution of Optical Transmission of Periodic Subwavelength Holes in a Metal Film. Phys. Rev. Lett. 2001, 86, 1110. [CrossRef] [PubMed]

9. Petronijevic, E.; Leahu, G.; Mussi, V.; Sibilia, C.; Bovino, F.A. Photoacoustic technique for the characterization of plasmonic properties of 2D periodic arrays of gold nanoholes. AIP Adv. 2017, 7, 025210. [CrossRef]

10. Schäferling, M.; Dregely, D.; Hentschel, M.; Giessen, H. Tailoring enhanced optical chirality: Design principles for chiral plasmonic nanostructures. Phys. Rev. X 2012, 2, 031010. [CrossRef]

11. Valev, V.K.; Baumberg, J.J.; Sibilia, C.; Verbiest, T. Chirality and chiroptical effects in plasmonic nanostructures: Fundamentals, recent progress, and outlook. Adv. Mater. 2013, 25, 2517-2534. [CrossRef]

12. Hentschel, M.; Schäferling, M.; Duan, X.; Giessen, H.; Liu, N. Chiral plasmonics. Sci. Adv. 2017, 3, e1602735. [CrossRef]

13. Caridad, J.M.; McCloskey, D.; Donegan, J.F.; Krstić, V. Controllable growth of metallic nano-helices at room temperature conditions. Appl. Phys. Lett. 2014, 105, 233114. [CrossRef]

14. Esposito, M.; Tasco, V.; Cuscuna, M.; Todisco, F.; Benedetti, A.; Tarantini, I.; De Giorgi, M.; Sanvitto, D.; Passaseo, A. Nanoscale 3D Chiral Plasmonic Helices with Circular Dichroism at Visible Frequencies. ACS Photonics 2015, 2, 105-114. [CrossRef]

15. Caridad, J.M.; Winters, S.; McCloskey, D.; Duesberg, G.S.; Donegan, J.F.; Krstić, V. Control of the plasmonic near-field in metallic nanohelices. Nanotechnology 2018, 29, 325204. [CrossRef] [PubMed]

16. Plum, E.; Fedotov, V.A.; Schwanecke, A.S.; Zheludev, N.I.; Chen, Y. Giant optical gyrotropy due to electromagnetic coupling. Appl. Phys. Lett. 2007, 90, 223113. [CrossRef]

17. Yang, S.; Liu, Z.; Yang, H.; Jin, A.; Zhang, S.; Li, J.; Gu, C. Intrinsic Chirality and Multispectral Spin-Selective Transmission in Folded Eta-Shaped Metamaterials. Adv. Opt. Mater. 2020, 8, 1901448. [CrossRef]

18. Leahu, G.; Petronijevic, E.; Belardini, A.; Centini, M.; Sibilia, C.; Hakkarainen, T.; Koivusalo, E.; Rizzo Piton, M.; Suomalainen, S.; Guina, M. Evidence of Optical Circular Dichroism in GaAs-Based Nanowires Partially Covered with Gold. Adv. Opt. Mater. 2017, 5, 1601063. [CrossRef]

19. Petronijevic, E.; Leahu, G.; Belardini, A.; Centini, M.; Li Voti, R.; Hakkarainen, T.; Koivusalo, E.; Rizzo Piton, M.; Suomalainen, S.; Guina, M.; et al. Photo-Acoustic Spectroscopy Reveals Extrinsic Optical Chirality in GaAs-Based Nanowires Partially Covered with Gold. Int. J. Thermophys. 2018, 39, 45. [CrossRef]

20. Petronijevic, E.; Centini, M.; Belardini, A.; Leahu, G.; Hakkarainen, T.; Sibilia, C. Chiral near-field manipulation in Au-GaAs hybrid hexagonal nanowires. Opt. Express 2017, 25, 14148. [CrossRef]

21. Gorkunov, M.V.; Ezhov, A.A.; Artemov, V.V.; Rogov, O.Y.; Yudin, S.G. Extreme optical activity and circular dichroism of chiral metal hole arrays. Appl. Phys. Lett. 2014, 104, 221102. [CrossRef]

22. Gorkunov, M.V.; Dmitrienko, V.E.; Ezhov, A.A.; Artemov, V.V.; Rogov, O.Y. Implications of the causality principle for ultra-chiral metamaterials. Sci. Rep. 2015, 5, 9273. [CrossRef]

23. Kondratov, A.V.; Gorkunov, M.V.; Darinskii, A.N.; Gainutdinov, R.V.; Rogov, O.Y.; Ezhov, A.A.; Artemov, V.V. Extreme optical chirality of plasmonic nanohole arrays due to chiral Fano resonance. Phys. Rev. B 2016, 93, 195418. [CrossRef] 
24. Kuppe, C.; Williams, C.; You, J.; Collins, J.T.; Gordeev, S.N.; Wilkinson, T.D.; Panoiu, N.; Valev, V.K. Circular Dichroism in Higher-Order Diffraction Beams from Chiral Quasiplanar Nanostructures. Adv. Opt. Mater. 2018, 6, 1800098. [CrossRef]

25. Arteaga, O.; Maoz, B.M.; Nichols, S.; Markovich, G.; Kahr, B. Complete polarimetry on the asymmetric transmission through subwavelength hole arrays. Opt. Express 2014, 22, 13719-13732. [CrossRef]

26. Petronijevic, E.; Ali, H.; Zaric, N.; Belardini, A.; Leahu, G.; Cesca, T.; Mattei, G.; Andreani, L.C.; Sibilia, C. Chiral effects in low-cost plasmonic arrays of elliptic nanoholes. Opt. Quantum Electron. 2020, 52, 176. [CrossRef]

27. Petronijevic, E.; Leahu, G.; Li Voti, R.; Belardini, A.; Scian, C.; Michieli, N.; Cesca, T.; Mattei, G.; Sibilia, C. Photo-acoustic detection of chirality in metal-polystyrene metasurfaces. Appl. Phys. Lett. 2019, 114, 053101. [CrossRef]

28. Petronijevic, E.; Belardini, A.; Leahu, G.; Cesca, T.; Scian, C.; Mattei, G.; Sibilia, C. Circular dichroism in low-cost plasmonics: 2D arrays of nanoholes in silver. Appl. Sci. 2020, 10, 1316. [CrossRef]

29. Ai, B.; Luong, H.M.; Zhao, Y. Chiral nanohole arrays. Nanoscale 2020, 12, 2479-2491. [CrossRef]

30. Cao, Z.L.; Ong, H.C. Determination of coupling rate of light emitter to surface plasmon polaritons supported on nanohole array. Appl. Phys. Lett. 2013, 102, 241109. [CrossRef]

31. Cao, Z.L.; Ong, H.C. Study of the momentum-resolved plasmonic field energy of Bloch-like surface plasmon polaritons from periodic nanohole array. Opt. Express 2017, 25, 30626-30635. [CrossRef]

32. Michieli, N.; Kalinic, B.; Scian, C.; Cesca, T.; Mattei, G. Emission Rate Modification and Quantum Efficiency Enhancement of Er3+ emitters by Near-Field Coupling with Nanohole Arrays. ACS Photonics 2018, 5, 2189-2199. [CrossRef]

33. Lumerical Solutions, Inc. Available online: http://www.lumerical.com/tcad-products/fdtd/ (accessed on 1 April 2021).

34. Petronijevic, E.; Centini, M.; Cesca, T.; Mattei, G.; Bovino, F.; Sibilia, C. Control of Au nanoantenna emission enhancement of magnetic dipolar emitters by means of $\mathrm{VO}_{2}$ phase change layers. Opt. Express 2019, 27, 24260. [CrossRef]

35. Hakkarainen, T.; Petronijevic, E.; Rizzo Piton, M.; Sibilia, C. Demonstration of extrinsic chirality of photoluminescence with semiconductor-metal hybrid nanowires. Sci. Rep. 2019, 5040. [CrossRef]

36. Petronijevic, E.; Belardini, A.; Cesca, T.; Scian, C.; Mattei, G.; Sibilia, C. Rich near-infrared chiral behavior in diffractive metasurfaces. Phys. Rev. Appl. 2021, 16, 014003.

37. Petronijevic, E.; Belardini, A.; Leahu, G.; Hakkarainen, T.; Rizzo Piton, M.; Koivusalo, E.; Sibilia, C. Broadband optical spin dependent reflection in self-assembled GaAs-based nanowires asymmetrically hybridized with Au. Sci. Rep. 2021, 11, 4316. [CrossRef] [PubMed]

38. Liu, X.; Yuan Lei, D. Simultaneous excitation and emission enhancements in upconversion luminescence using plasmonic double-resonant gold nanorods. Sci. Rep. 2015, 5, 15235. [CrossRef] [PubMed]

39. Petronijevic, E.; Sibilia, C. All-optical tuning of EIT-Like dielectric metasurfaces by means of chalcogenide phase change materials. Opt. Express 2016, 24, 30411. [CrossRef]

40. Bauch, M.; Toma, K.; Toma, M.; Zhang, Q.; Dostalek, J. Plasmon-Enhanced Fluorescence Biosensors: A Review. Plasmonics 2014, 9, 781-799. [CrossRef] [PubMed]

41. Pirruccio, G.; Lozano, G.; Zhang, Y.; Rodriguez, S.R.K.; Gomes, R.; Hens, Z.; Gomez Rivas, J. Coherent absorption and enhanced photoluminescence in thin layers of nanorods. Phys. Rev. B 2012, 85, 165455. [CrossRef]

42. Ayala-Orozco, C.; Liu, J.G.; Knight, M.W.; Wang, Y.; Day, J.K.; Nordlander, P.; Halas, N.J. Fluorescence Enhancement of Molecules Inside a Gold Nanomatryoshka. Nano Lett. 2014, 14, 2926-2933. [CrossRef]

43. Pirruccio, G.; Ramezani, M.; Rahimzadeh-Kalaleh Rodriguez, S.; Gómez Rivas, J. Coherent Control of the Optical Absorption in a Plasmonic Lattice Coupled to a Luminescent Layer. Phys. Rev. Lett. 2016, 116, 103002. [CrossRef] [PubMed]

44. Djalalian-Assl, A. Dipole Emission to Surface Plasmon-Coupled Enhanced Transmission in Diamond Substrates with Nitrogen Vacancy Center- Near the Surface. Photonics 2017, 4, 10. [CrossRef]

45. Ferraro, A.; Zografopoulos, D.C.; Verschuuren, M.A.; de Boer, D.K.G.; Kong, F.; Urbach, H.P.; Beccherelli, R.; Caputo, R. Directional Emission of Fluorescent Dye-Doped Dielectric Nanogratings for Lighting Applications. ACS Appl. Mater. Interfaces 2018, 10, 24750-24757. [CrossRef] [PubMed]

46. Petronijevic, E.; Sandoval, E.M.; Ramezani, M.; Ordóňez-Romero, C.L.; Noguez, C.; Bovino, F.A.; Sibilia, C.; Pirruccio, G. Extended Chiro-optical Near-Field Response of Achiral Plasmonic Lattices. J. Phys. Chem. C 2019, 123, 23620-23627. [CrossRef] 